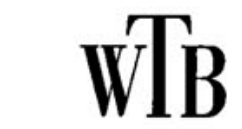

BAND 53

Harry Paul

Lasertheorie I

Mit 24 Abbildungen

AKADEMIE-VERLAG - B ERLIN

PERGAMON PRESS - OXFORD

VIEWEG \& SOHN - BRAUNSCHWEIG 


\section{Reihe MATHEMATIK UND PHYSIK}

Herausgeber:

Prof. Dr. rer. nat. habil. G. Heber, Leipzig

Prof. Dr. phil. habil. W. Holzmüller, Leipzig

Prof. Dr. phil. habil. A. Lösche, Leipzig

Prof. Dr. phil. habil. H. Reichardt, Berlin

Prof. Dr. phil. habil. J. Schintlmeister, Dresden

Prof. Dr. phil. habil. K. Schröder, Berlin

Prof. Dr. phil. habil. K. Schröter, Berlin

Prof. Dr. rer. nat. habil. H.-J. Treder, Potsdam

Verantwortlicher Herausgeber dieses Bandes:

Prof. Dr. G. Heber

Verfasser:

Dr. H. Paul

Institut für spezielle Probleme der theoretischen Physik

der Deutschen Akademie der Wissenschaften zu Berlin

Copyright 1969 by Akademie -Verlag GmbH, 108 Berlin Lizenznummer : $202 \cdot 100 / 423 / 69$

Gesamtherstellung: VEB Druckhaus „Maxim Gorki“, 74 Altenburg

Bestellnummer: Akademie-Verlag 7053 - ES 18 B 6/7

Pergamon Press 080068448

Vieweg \& Sohn 6053

Printed in German Democratic Republic 


\section{Inhaltsverzeichnis}

Vorwort . . . . . . . . . . . . . 5

1. Die grundlegenden Prozesse im Laser . . . . . . . . 9

1.1. Einleitung. Das Laserprinzip . . . . . . . . . . . 9

1.2. Emission und Absorption . . . . . . . . . . . . . 12

1.3. Pumpen ............... . . 36

1.4. Rückkopplung . . . . . . . . . . . . . . . 41

2. Elektromagnetische Felder in offenen optischen Resonatoren . . . . . . . . . . . . 45

2.1. Heuristische Uberlegungen . . . . . . . . . 45

2.2. Strenge Formulierung des Eigenwertproblems . . . . . 56

2.3. Ergebnisse numerischer Rechnungen . . . . . . . 61

3. Bilanzgleichungen für den Laserproze $B$. . . . . . 70

3.1. Aufstellung der Gleichungen . . . . . . . . . . . . 70

3.2. Lösung im stationären Fall . . . . . . . . . . . . 79

3.3. Einschwingvorgänge . . . . . . . . . . . . . 83

4. Die Lambsche Theorie des Gaslasers. . . . . . . . . 88

4.1. Das Verhalten eines Atoms in einem äußeren Feld . . 88

4.2. Die Grundkonzeption der Theorie . . . . . . . . 110

4.3. Die Grundgleichungen . . . . . . . . . . . . . . 125

4.4. Genäherte Lösung der ScHRöDINGER-Gleichung . . . . 139

4.5. Berechnung der Polarisation in erster Näherung . . . 141

4.6. Schwellenbedingung und Frequenzverschiebung . . . 154

4.7. Der Ein-Frequenz-Betrieb des Lasers . . . . . . . . 157

4.8. Das „Löcher-Brennen“ („,hole burning“) . . . . . . 165

4.9. Gleichzeitige Anregung zweier Eigenschwingungen . . 167

4.10. Frequenzsynchronisation (,frequency locking“) . . . 176

Sachverzeichnis . . . . . . . . . . . . . 182 
